Conclusion: The new axSpA-SQUASH resulted in improved content validity and measurement properties. It seems the most appropriate questionnaire and can be used to assess daily physical activity in patients with axSpA.

References:

[1] Van Der Heijde D et al. Ann Rheum Dis. 2017;76:978-91.

[2] Arends S et al. Arthritis Res Ther. 2013;15:R99.

Acknowledgments: We thank the ASAS for the reserch grant that supported this work.

Disclosure of Interests: Marlies Carbo: None declared, Davy Paap: None declared, Fiona Maas: None declared, Anna Jetske Baron: None declared, Laura van Overbeeke: None declared, Mark Siderius: None declared, Freke Wink Consultant of: Abbvie, Janssen, Hendrika Bootsma Grant/research support from: Unrestricted grants from Bristol-Myers Squibb and Roche, Consultant of: Consultant for Bristol-Myers Squibb, Roche, Novartis, Medimmune, Union Chimique Belge, Speakers bureau: Speaker for Bristol-Myers Squibb and Novartis., Suzanne Arends Grant/research support from: Grant/research support from Pfizer, Anneke Spoorenberg: None declared DOI: 10.1136/annrheumdis-2020-eular.1152

\section{FRI0569 SERUM AMYLOID A: ASSESSMENT OF REFERENCE VALUE AND COMPARISON OF SERUM CONCENTRATION IN HEALTHY SUBJECTS AND PATIENTS WITH BEHÇET SYNDROME}

T. Carbone $^{1}$, M. C. Padula ${ }^{1}$, V. Pafundi ${ }^{2}$, C. Schievano ${ }^{3}$, N. Lascaro ${ }^{1}$, A. Padula $^{1}$, P. Leccese ${ }^{1}$, S. D'angelo ${ }^{1} .{ }^{1} I R e L$ - Rheumatology Institute of Lucania - San Carlo Hospital, Potenza, Italy; ${ }^{2}$ Immunopathology Laboratory - San Carlo Hospital, Potenza, Italy; ${ }^{3}$ Innovative Statistical Research, Padova, Italy

Background: Serum amyloid A (SAA) is a family of acute-phase reactants. The rise of SAA concentration in blood circulation is a clinical marker of active inflammation in several auto-inflammatory diseases, including Behçet syndrome (BS). Despite its practical and analytical advantages, SAA measurement by ELISA has been mainly used as a research tool rather than for the routine laboratory testing due to the lack of a robust reference data in the literature.

Objectives: Using the recommended procedures of the International Federation of Clinical Chemistry and Laboratory Medicine (IFCC), we aimed to develop the SAA reference interval for a well-defined Italian healthy population (HC). Secondly, we compared the SAA serum concentration between $\mathrm{HC}$ and patients with BS.

Methods: Sera specimens were collected from adult healthy blood donors after rule out the exclusion criteria (inflammatory disorders, ongoing infections, pregnancy and breastfeeding, obesity, using oral contraceptives, use of any medication, or consumed of alcohol), and from unselected BS patients fulfilling the International Study Group (ISG) classification criteria. Serum SAA concentrations were detected and quantified with a commercial solid phase sandwich enzyme-linked immunosorbent assay (Human SAA ELISA kit, IBL International $\mathrm{GmbH}$, Hamburg, Germany) used on automated analyzer (Immunomat, SERION Diagnostic, Alifax, Polverara (PD), Italy) according to the manufacturer's protocol. Statistical analysis and data normalization of HC SAA values were carried out to determine the reference cut off. In the second step of the study, $\mathrm{HC}$ and BS patients were stratified in two groups according to the cut-off value.

Results: We recruited $141 \mathrm{HC}$ ( $84 \mathrm{M}$ and $57 \mathrm{~F}$; mean age, $44.5 \pm 13.2$ years) and 63 BS patients (39M and $24 \mathrm{~F}$ mean age, $45.3 \pm 13.2$ years) assayed for SAA. The reference cut-off was calculated as $225 \mathrm{ng} / \mathrm{ml}$. No statistically significant differences were found between males and females when SAA means were compared, suggesting that not gender-partitioned reference range is recommended for this analyte. After the stratification according to the cut-off value (group 1: < $225 \mathrm{ng} / \mathrm{ml}$ and group 2: $>225 \mathrm{ng} / \mathrm{ml})$, we found $53 / 63(84.1 \%)$ BS patients and $133 / 141$ (94.3\%) HC with concentration less than cut-off value, respectively. We identified 10/63 (15.9\%) BS patients and 8/141 (5.7\%) HC within the second group. The difference was statistically significant $(p=0.0177$; OR: $3.14,95 \% \mathrm{Cl}$ : 1.17-3.38).

Conclusion: This study allowed to define a widely accepted reference cut-off for the SAA detected by ELISA, responding to an unmet need of laboratory medicine. We found a statistically significant higher frequency of BS patients compared with $\mathrm{HC}$ when SAA values is higher than cut-off $(225 \mathrm{ng} / \mathrm{ml})$. This preliminary data could add significant information for better clarify the role of SAA as biomarker of inflammation and in guidance of clinical practice. Further studies will be required to stratify SAA values in relation to disease activity of BS.

Disclosure of Interests: Teresa Carbone: None declared, Maria Carmela Padula: None declared, Vito Pafundi: None declared, Carlo Schievano: None declared, Nancy Lascaro: None declared, Angela Padula: None declared, Pietro Leccese:
None declared, Salvatore D’Angelo Consultant of: AbbVie, Biogen, BMS, Celgene, Eli Lilly, MSD, Novartis, and UCB, Speakers bureau: AbbVie, BMS, Celgene, Eli Lilly, Novartis, Pfizer, and Sanofi

DOI: 10.1136/annrheumdis-2020-eular.6357

\section{FRI0570 \\ IDENTIFICATION OF OSTEOPONTIN/SECRETED PHOSPHOPROTEIN 1 AS A BIOMARKER FOR PSORIATIC ARTHRITIS}

F. Abji ${ }^{1}$, R. Machhar ${ }^{1}$, K. Liang ${ }^{2}$, J. Ye ${ }^{1}$, K. Oikonomopoulou ${ }^{1}$, V. Chandran ${ }^{1}$. ${ }^{1}$ University Health Network, Toronto, Canada; ${ }^{2}$ University of Waterloo, Waterloo, Canada

Background: Early diagnosis of psoriatic arthritis (PsA) can be facilitated by appropriate referral of psoriasis patients to rheumatologists. Soluble biomarkers for PsA may help screen psoriasis patients for PsA.

Objectives: To identify novel biomarkers for PsA by investigating serum levels of candidate biomarkers identified through proteomic analysis of synovial fluid (SF) and skin biopsies and literature review.

Methods: We first (discovery phase) identified markers using: i) proteomic analysis of $\mathrm{SF}^{1}$, ii) proteomic analysis of skin biopsies ${ }^{2}$, and iii) literature review. In verification phase 1 , we measured serum levels of the selected potential protein markers, using commercially available ELISA kits, to identify differentially expressed markers in healthy controls and patients with PsA ( $\geq 3$ swollen joints not treated with biologics) and psoriasis without PsA (PsC; matched with PsA patients on age, sex and psoriasis duration) (100 subjects each group). In verification phase 2, using less strict criteria (no restriction on pharmacotherapy or disease activity) and larger sample size, we confirmed the association with PsA of markers identified in phase 1 using samples from 200 patients each with PsA and PsC. Statistical methods used included descriptive statistics, t-tests and logistic regression.

Results: The discovery phase identified the following 31 markers for testing in verification phase 1- hsCRP, MMP3, CD5L, M2BP, MPO, ITGB5, DKK1, FGF23, IL-6, IL-1 $\beta$, leptin, osteocalcin, OPG, OPN, SOST, TNFa, adiponectin, periostin, RANKL, YKL40, KLK6, KLK8, CS846, C2C, CPII, TNFSF14, COMP, ALP, CXCL10, S100A8/A9 and DEFA. The following 21 markers remained differentially upregulated in PSA after testing in verification phase 1- hsCRP, MMP-3, M2BP, ITGB5, leptin, OPG, OPN, SOST,TNFa, periostin, RANKL, YKL40, KLK8, C2C, CPII, TNFSF14, COMP, ALP, CXCL10, S100A8/A9 and DEFA. Univariate logistic regression analyses adjusted for age, sex, and disease duration confirmed the association between hsCRP, OPN, S100A8/ $\mathrm{A}$, OPG and the ratio $\mathrm{CPII} / \mathrm{C} 2 \mathrm{C}$ in verification phase 2 . Multivariate logistic regression demonstrated that hsCRP and OPN (both $p<0.001$ ) are independently associated with PsA.

Conclusion: OPN, a cytokine involved in enhancing production of IFN $\gamma$ and IL-12, reducing production of IL-10 and promoting attachment of osteoclasts to mineralized bone matrix, is a potential biomarker of PsA.

References:

[1] Cretu D, Prassas I, Saraon P, Batruch I, Gandhi R, Diamandis EP, Chandran V. Identification of psoriatic arthritis mediators in synovial fluid by quantitative mass spectrometry. Clin Proteomics. 2014 Jul 1;11(1):27.

[2] Cretu D, Liang K, Saraon P, Batruch I, Diamandis EP, Chandran V. Quantitative tandem mass-spectrometry of skin tissue reveals putative psoriatic arthritis biomarkers. Clin Proteomics. 2015 Jan 13;12(1):1.

Disclosure of Interests: Fatima Abji: None declared, Rohan Machhar: None declared, Kun Liang: None declared, Justine Ye: None declared, Katerina Oikonomopoulou: None declared, Vinod Chandran Grant/research support from: Abbvie, Celgene, Consultant of: Abbvie, Amgen, Bristol-Myers Squibb Celgene, Eli Lily, Janssen, Novartis, Pfizer, UCB, Employee of: Spouse employed by Eli Lily

DOI: 10.1136/annrheumdis-2020-eular.4536

\section{FRI0571 1 THE DIAGNOSTIC VALUE OF KL-6 IN RHEUMATOID ARTHRITIS ASSOCIATED WITH INTERSTITIAL LUNG DISEASE IN XINJIANG OF CHINA}

G. Chen ${ }^{1}$, L. Wu ${ }^{1} .{ }^{1}$ People's Hospital of the Xinjiang Uygur Autonomous Region, Urumqi, China

Background: Rheumatoid arthritis (RA) is a systemic inflammatory disease.Many researchers have observed that extra articular organs were highly involved in RA patients. The most common extra-articular manifestations were pulmonary involvement.Serum levels of KL-6 have been reported to be elevated in various ILD such as idiopathic pulmonary fibrosis, collagen vascular disease associated interstitial pneumonias, and other interstitial lung disorders. However, little is known regarding 
the usefulness of this biomarker in connective tissue diseases related interstitial lung diseases(CTD-ILD). Especially, the diagnostic value of KL-6 in interstitial lung disease associated with rheumatoid arthritis(RA-ILD) still has a dispute.

Objectives: To assess the diagnosis of the serum Krebs von den Lungen-6(KL-6) for RA-ILD patients in Xinjiang of China.

Methods: This retrospective study included 184 patients with RA in who visited the department of rheumatology and immunology of People's Hospital of Xinjiang Uygur Autonomous Region between January, 2015 and December, 2019.The patients were divided into RA-ILD group $(n=95)$ and RA group $(n=89)$ according to the presence of ILD. Serum KL-6 concentration $(\mathrm{U} / \mathrm{mL})$ was measured using the chemiluminescent enzyme immunoassay kit.

Results: The mean age $(p<0.001)$ and median value of CCP $(p=0.006)$ were significantly higher in the RA-ILD group.RA-ILD group had elevated serum KL-6 levels compared to RA group $[447(281,687) \mathrm{U} / \mathrm{ml}$ vs $195(151.5,265.5) \mathrm{U} / \mathrm{ml}](\mathrm{p}<$ 0.001)(figure 1).According to the Receiver Operating Characteristic Curve (ROC) analysis, the area under the curve was of 0.879 and the optimal cut-off value of serum KL-6 to discriminate the presence of ILD was $277 \mathrm{U} / \mathrm{ml}$, with sensitivity of $77.9 \%$, specificity of $79.8 \%$ (figure 2 ).

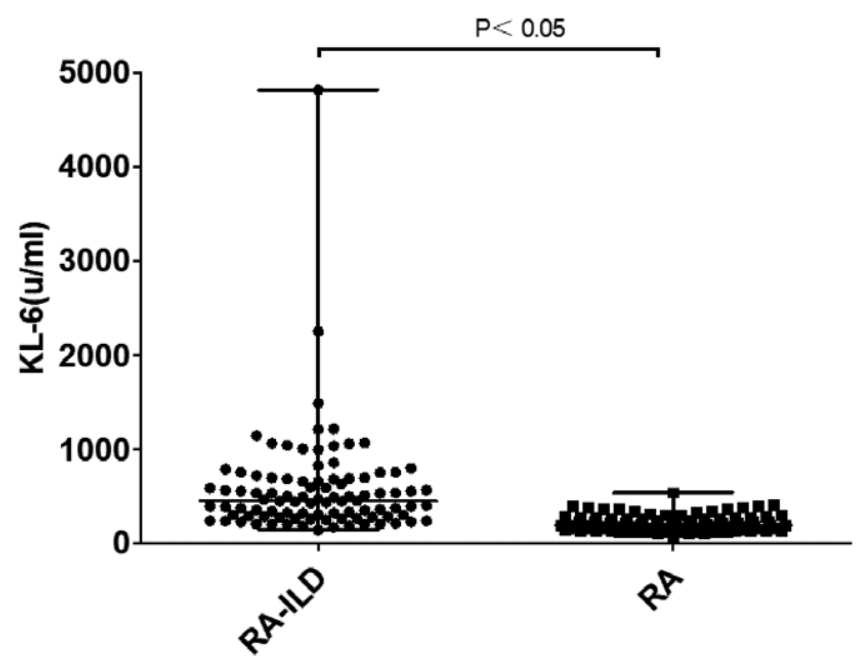

Figure 1. Comparison of serum $\mathrm{KL}-6$ concentrations in RA-ILD group and RA group.

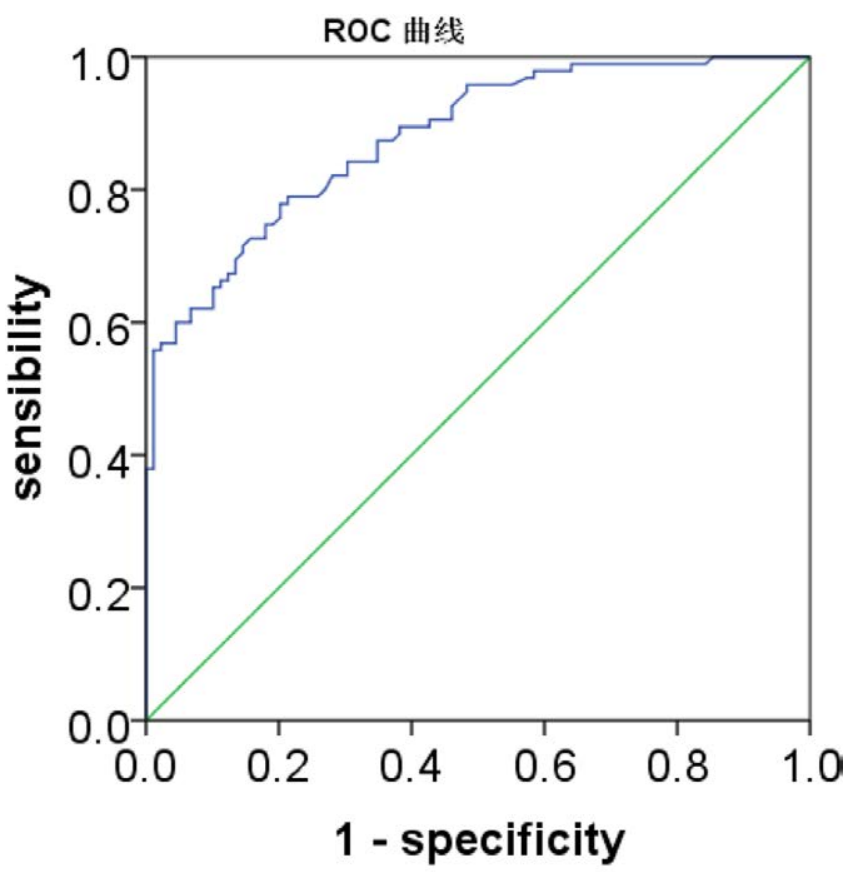

结生成的对角段。

Figure. 2. Receiver-operating characteristic curve(ROC) of KL-6 for the diagnosis of RA-ILD
Conclusion: The present study confirms that KL- 6 is a biological marker which is associated with RA-ILD. Furthermore, Patients with RA who are older and have a higher value of CCP are more likely to develop ILD.

References:

[1] Anaya JM, Diethelm L, Ortiz LA et al (1995) Pulmonary involvement in rheumatoid arthritis. Semin Arthritis Rheum 24:242-254

[2] Bonella F, Costabel U (2014) Biomarkers in connective tissue disease-associated interstitial lung disease. Semin Respir Crit Care Med 35:181-200

[3] Doishita S, Inokuma S, Asashima H et al (2011) Serum KL-6 level as an indicator of active or inactive interstitial pneumonitis associated with connective tissue diseases. Intern Med 50: 2889-2892

Disclosure of Interests: None declared

DOI: 10.1136/annrheumdis-2020-eular.5736

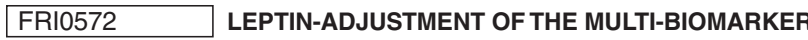 DISEASE ACTIVITY (MBDA) SCORE REDUCES THE INFLUENCE OF ADIPOSITY}

J. Baker ${ }^{1}$, J. Curtis ${ }^{2}$, D. Chernoff ${ }^{3}$, M. George ${ }^{1} .{ }^{1}$ University of Pennsylvania Perelman School of Medicine, Philadelphia, United States of America; ${ }^{2}$ University of Alabama at Birmingham, Birmingham, United States of America; ${ }^{3}$ Myriad Autoimmune (consultant), South San Francisco, United States of America

Background: Obesity and excess adiposity influence inflammatory markers and bias disease activity assessment, especially among women. A multi-biomarker disease activity (aMBDA) score has been developed to account for the effects of age, sex and adiposity (leptin) and improves prediction of radiographic damage progression.

Objectives:

1) Determine if the adjusted measure demonstrates a reduced association with adiposity.

2) Assess the impact of the leptin-adjustment on the score over the range of adiposity.

3) Assess relationships between MBDA scores and clinical disease activity.

Methods: Patients with rheumatoid arthritis (RA), ages 18-75 years, completed whole-body dual-energy $x$-ray absorptiometry to quantify fat mass indices (FMI, kg/m ${ }^{2}$ ). Age-, sex-, and race-specific Z-Scores were calculated based on the distributions in a healthy reference population. Disease activity was assessed with the CDAI and swollen joint count (SJC). Baseline van de Heijde-Sharpe (vdHS) scores were determined by a radiologist. MBDA assays were performed on stored serum samples. Descriptive statistics described relationships between the FMI Z-Score and the MBDA and the aMBDA. Clinical disease activity, SJC, and radiographic damage were also compared across MBDA score categories.

Results: Of 104 participants (50\% female), the mean (SD) age was 56.1 (12.5) and mean BMI was 28.8 (6.9) (Table 1). The unadjusted MBDA score was strongly associated with BMI among women (Women: Rho=0.46 [p< 0.001]; Men: Rho=0.12 ), while the aMBDA was not associated with $\mathrm{BMI}$ in women and was inversely correlated in men (Women: Rho=0.17; Men: Rho=-0.32 [p=0.02]). The unadjusted MBDA score was also strongly associated with FMI Z-Score among women (Figure; Women: Rho $=0.42$ [p=0.002]; Men: Rho $=-0.10 ; p=0.01)$. The aMBDA was not significantly associated with FMI Z-Score (Female: Rho=0.17; Male: Rho=0.26). Leptin-adjustment reduced the MBDA score in the highest quartile of FMI in women but not men, and increased the MBDA score in the lowest FMI quartiles in both women and men; these patients in the lowest FMI quartile had the highest median SJC ( $p=0.05$ for men, $p=0.78$ for women; Figure). The aMBDA reclassified 4 women (8\%) and 9 men (17\%) into higher disease activity categories and 2 women (4\%) and 2 men (4\%) into lower categories. CDAI, SJC, and radiographic scores were similar across activity categories for the unadjusted MBDA score and aMBDA (Table 2).

Table 1. Baseline Characteristics.

\begin{tabular}{lcc}
\hline & Men & Women \\
\hline $\mathrm{N}$ & 52 & 52 \\
Age (yrs) & $59.1(11.5)$ & $53.0(12.8)$ \\
Black, N (\%) & $13(25 \%)$ & $19(36 \%)$ \\
BMI & $27.3(5.4)$ & $30.3(8.0)$ \\
FMI Z-Score & $-0.28(1.3)$ & $0.05(1.1)$ \\
DAS28(CRP) & $3.09(1.13)$ & $3.21(1.24)$ \\
Disease Duration & $11.4(10.9)$ & $11.6(11.9)$ \\
CRP, mg/dL & $0.8(0.5,1.2)$ & $0.8(0.5,1.4)$ \\
CCP Positive, N (\%) & $45(87 \%)$ & $40(78 \%)$ \\
vdHS (N=93) & $13(4,73)$ & $10.5(2,47)$ \\
HAQ & $0.71(0.59)$ & $0.83,(0.67)$ \\
MBDA & $40.0(13.8)$ & $42.1(16.6)$ \\
aMBDA & $43.6(13.4)$ & $42.1(15.3)$ \\
Leptin, ng/mL & $15.1(21.5)$ & $48.9(41.5)$ \\
\end{tabular}

